\title{
Human papillomavirus (HPV) genotypes among HIV-infected and HIV-uninfected women in Mozambique
}

\author{
Massimo Magnano San Lio ${ }^{1,2^{*}}$, Ivo Marchetti ${ }^{3}$, Carla Carrilho ${ }^{4}$, Maria Pia Cioni ${ }^{3}$, Giovanni Guidotti ${ }^{3,1}$, \\ Cristina Moscatelli ${ }^{3,1}$, Fabio Taponeco ${ }^{6}$, Elias Suizane F Walle ${ }^{5}$, Ines Zimba ${ }^{7}$, Generoso Bevilacqua ${ }^{3}$ \\ From $16^{\text {th }}$ International Symposium on HIV and Emerging Infectious Diseases \\ Marseille, France. 24-26 March 2010
}

\section{Background}

The objective of this study was to determine the prevalence of HPV infection and related genotypes in a group of HIV-infected women and in a control group of HIVuninfected women. To our knowledge, it is the first study such study conducted in Mozambique.

\section{Methods}

The study was conducted in a public health center in Maputo. It was performed in the context of the Drug Resource Enhancement against AIDS and Malnutrition (DREAM) program, managed by the Community of San$t^{\prime}$ Egidio within the national health system in collaboration with Ministry of Health. This is a prospective, two-arm, observational study. The first arm includes HIV- infected women while the second arm involves a control group of HIV-uninfected women. The enrollment period lasted 6 months (August 2007- January 2008). The observation period was 6 months. HPV detection genotyping was performed using CLINICAL ARRAYS $^{\oplus}$ (GENOMICA SAU). HIV was detected with b-DNA assay (HIVç1 RNA 3.0, Bayer Health Care).

\section{Results}

The study involved 191 participants: $141 \mathrm{HIV}$-infected and 50 HIV-uninfected women. HPV was found in 126/141 (89.4\%) of those HIV infected and in 33/50 (66\%) of HIVuninfected subjects $(p<0.001)$. In HIV-infected women there were 94/126 (74.6\%) HPV-multiple-infections while in HIV-uninfected women there were $22 / 33$ (66.6\%) $(p=0.375)$. Twenty-nine distinct HPV types were

* Correspondence: massimo.magnano@gmail.com

${ }^{1}$ Community of Sant'Egidio, Drug Resources Enhancement against AIDS and Malnutrition (DREAM) Program, Roma, Italy identified among the $141 \mathrm{HIV}$-infected women, of which 16 viral types were classified as cancer high-risk or probable high-risk viruses. The most common types identified were HPV types $58(12.1 \%, p<0.001), 16(10.7 \%, p=$ $0.06), 61(8.4 \%, p<0.05), 53(7.9 \%, p=0.55)$ and $6(7.6 \%$, $p=0.41)$. Nineteen distinct HPV genotypes were identified among the $50 \mathrm{HIV}$-uninfected women. Ten types were of high-risk or probable high-risk. The most common types identified in HIV-uninfected subjects were HPV types 53 (10.4\%), 6 (9.1\%), 16 (9.1\%), 18 (9.1\%) and 66 (7.8\%).

\section{Discussion}

HPV infection was mainly associated with HIV positive status. HPV multiple infections were high in the population studied, independently of HIV status. HPV Genotypes are different in the two groups. The HPV types identified are partially different from those more commonly identified in Western countries.

\section{Author details \\ ${ }^{1}$ Community of Sant'Egidio, Drug Resources Enhancement against AIDS and Malnutrition (DREAM) Program, Roma, Italy. ${ }^{2}$ Azienda U.S.L. Roma F Civitavecchia, Italy. ${ }^{3}$ Division of Surgical, Molecular and Ultrastructural Pathology, University of Pisa and University Hospital of Pisa, Pisa, Italy. ${ }^{4}$ Department of Pathology, Universidade Eduardo Mondlane, Hospital Central de Maputo, Ministério de Saúde, Maputo, Mozambique. ${ }^{5}$ Department of Gynecology, Hospital Central de Maputo, Ministério de Saúde, Maputo, Mozambique. ${ }^{6}$ Department of Gynecology, University Hospital of Pisa, Pisa, Italy. ${ }^{7}$ DREAM Health Center of Benfica, Maputo, Mozambique.}

Published: 11 May 2010

doi:10.1186/1742-4690-7-S1-P2

Cite this article as: Lio et al:: Human papillomavirus (HPV) genotypes among HIV-infected and HIV-uninfected women in Mozambique. Retrovirology 2010 7(Suppl 1):P2. 